\title{
Diazoxide-responsive hyperinsulinaemic hypoglycaemia in tyrosinaemia type 1
} \author{
Spyros Batzios ${ }^{2}$, Sebastian Kummer ${ }^{3}$ and Pratik Shah ${ }^{1, \dagger}$ \\ 1Endocrinology Department, 2Metabolic Department, Great Ormond Street Hospital for Children, NHS Foundation \\ Trust, London, UK, and '3Department of General Paediatrics, Neonatology and Paediatric Cardiology, University \\ Children's Hospital, Medical Faculty, Heinrich-Heine University, Duesseldorf, Germany

Ellada Sotiridou', Henrike Hoermann (133), Sommayya Aftab', Antonia Dastamani', Eva Thimm³, Louise Doodson',

Correspondence should be addressed to E Sotiridou Email

ellada.sotiridou@nnuh. nhs.uk

\section{Summary}

Tyrosinaemia type 1 (TT1) is a rare inherited disorder of amino acid metabolism typically presenting with liver failure and renal tubular dysfunction. We describe three individuals with TT1 and transient hyperinsulinaemic hypoglycaemia $(\mathrm{HH})$. Two siblings with TT1 and acute liver dysfunction were diagnosed with hyperinsulinaemic hypoglycaemia in the neonatal period. Both siblings were successfully treated with diazoxide/chlorthiazide and treatment was gradually weaned and stopped after 8 and 6 months of age respectively. The third patient presented with a neonatal liver failure with mild cholestasis, coagulopathy, fundus haemorrhages, vitamin A and E deficiency and hyperinsulinaemic hypoglycaemia. He maintained euglycaemia on high dose diazoxide (5-12 mg/kg/day) but developed pulmonary hypertension at 12 weeks of age. After discontinuation of diazoxide, he continued maintaining his blood glucose (BG) within the normal range. Although histological abnormalities of the pancreas including beta-cell hyperplasia are well documented, the exact mechanism of excessive insulin secretion in $\mathrm{TT} 1$ is not well understood. It may be related to the accumulation of toxic metabolites in the target organs including pancreas. Therefore, in patients with TT1 and persistent hypoglycaemia beyond the recovery of the acute liver failure, it is important to exclude hyperinsulinism which is usually transient and can be successfully treated with diazoxide and chlorothiazide. Further studies are required to determine which factors contribute to excessive insulin secretion in patients with TT1.

\section{Learning points:}

- Every child with TT1 should be monitored for signs and symptoms of hypoglycaemia and screened for HH at the time of real hypoglycaemia.

- If hypoglycaemic episodes persist even after improvement of liver function, hyperinsulinism should be suspected.

- Treatment with diazoxide is effective, however, children need to be monitored closely for possible side effects.

- The pathophysiological mechanism of hyperinsulinism in children with TT1 is not elucidated yet and further studies are required to determine which factors contribute to excessive insulin secretion in patients with TT1.

\section{Background}

Tyrosinaemia type 1 (TT1) is a rare autosomal recessively inherited metabolic disorder characterised by disruption of the last step of tyrosine metabolism. The resulting accumulation of toxic metabolites such as maleylacetoacetate, fumarylacetoacetate and succinylacetone leads to progressive liver disease, renal tubular dysfunction and porphyria-like crises (1). Hypoglycaemia is not uncommon, especially 
in the acute form of TT1, and could contribute to neurodevelopmental impairment observed later in life. In regard to aetiopathological mechanisms, hypoglycaemia was described to be associated with acute liver failure and reduced hepatic clearance of insulin (2). So far, only a few patients have been reported with hyperinsulinaemic hypoglycaemia $(\mathrm{HH})$ persisting beyond the recovery of the acute liver failure (3). Here we describe three cases of TT1 with confirmed transient $\mathrm{HH}$ persisting for several months following the acute liver failure recovery.

\section{Case reports}

Patients' clinical and biochemical findings are summarised in Table 1.

\section{Case 1}

The first patient was a female child born at term to consanguineous parents. She was diagnosed with elevated phenylalanine in newborn screening and liver failure, which triggered further metabolic investigations that revealed high plasma tyrosine levels indicative of TT1. Analysis of urine organic acids revealed elevated 4-hydroxyphenyllactate, 4-hydroxyphenylpyruvate and succinylacetone, consistent with TT1. She was commenced on a tyrosine- and phenylalanine-restricted diet and Nitisinone (NTBC, 2-(2-nitro4-trifluoromethylbenzoyl)1,3-cyclohexanedione) (1) which was well tolerated. Genetic testing has shown that the patient was homozygous for the pathogenic variant c.192G>T p.(Gln64His). Analysis of parental samples confirmed that both parents were heterozygous for the mutation. She was also found to be hypoglycaemic on Day 5 of her life and required intravenous glucose with a glucose infusion rate of up to $9 \mathrm{mg} / \mathrm{kg} / \mathrm{min}$. Hypoglycaemia screen revealed detectable insulin and inability to mobilise ketones and fatty acids confirming the diagnosis of $\mathrm{HH}$ (Table 1). Therefore she was commenced on diazoxide $(3 \mathrm{mg} / \mathrm{kg} /$ day) and chlorothiazide (7 mg/kg/day) on Day 10 of life and responded well. She was discharged home after a couple of weeks, fasting up to $6 \mathrm{~h}$ without supplemental carbohydrates and with normal blood glucose (BG) at the end of the fast. Hypoglycaemia persisted even after recovery of liver function. She continued to outgrow diazoxide and after 8 months age, diazoxide was stopped and she was able to fast for $12 \mathrm{~h}$ with normal BG at the end of the fast.

Table 1 Clinical and biochemical features of three subjects with TT1 and transient HI.

\begin{tabular}{l}
\hline \\
\hline Birth weight (grams) \\
Gestation (weeks) \\
Diagnosis of TT1 (days) \\
Neonatal screening \\
Urine organic acid analysis \\
(succinylacetone) \\
Genetic confirmation \\
Genotype \\
\\
Onset of initial hypoglycaemia \\
Hypoglycaemia screen \\
Lab glucose (3.5-5.5 mmol/L) \\
Insulin (<2 mU/L) \\
C-peptide ( pmol/L) \\
Non-esterified fatty acids \\
(1-2mmol/L) \\
-Hydroxybutyra (1-2mmol/L) \\
Lactate (0.7-2.1 mmol/L) \\
HI treatment initiated \\
Diazoxide dose \\
Diazoxide duration \\
Diuretics \\
Diuretics length \\
Pulmonary hypertension \\
Fasting tolerance \\
\end{tabular}

\begin{tabular}{l}
\hline Patient 1 \\
\hline 2700 (-2.11 SDS) \\
Term \\
Yes (Day 5) \\
Yes \\
Yes (Day 50) \\
Homozygous for pathogenic \\
variant c.192G $>$ Tp. \\
(Gln64His) in FAH-gene \\
Day 5 \\
$2.1 \mathrm{mmol} / \mathrm{L}$ (38 mg/dL) \\
$3.8 \mathrm{mU} / \mathrm{L}$ \\
$610 \mathrm{pmol} / \mathrm{L}$ \\
$<0.05 \mathrm{mmol} / \mathrm{L}$ \\
$0.22 \mathrm{mmol} / \mathrm{L}$ \\
$2.4 \mathrm{mmol} / \mathrm{L}$ \\
\\
Day 10 \\
3 mg/kg/day \\
8 months \\
Chlorothiazide (7 mg/kg/day \\
in two divided doses). \\
8 months \\
No \\
12 hours off diazoxide at the \\
age of 8 months \\
\end{tabular}

\begin{tabular}{l} 
Patient 2 \\
\hline 2280 (-1.5 SDS) \\
36 \\
Yes (Day 5$)$ \\
Yes \\
Yes (Day 28 ) \\
Homozygous for pathogenic \\
variant c.192G Tp. \\
(Gln64His) in FAH-gene \\
Day 14 \\
1.9 mmol/L (34 mg/dL) \\
4.6 mU/L \\
348 pmol/L \\
0.05 mmol/L \\
0.05 mmol/L \\
1.1 mmol/L \\
Day 21 \\
3 mg/kg/day \\
6 months \\
Chlorothiazide (7 mg/kg/day \\
in two divided doses). \\
6 months \\
No \\
12 hours off diazoxide \\
at the age of 6 months \\
\end{tabular}

\begin{tabular}{l}
\hline Patient 3 \\
\hline 2530 (-1.03 SDS) \\
$36+4$ \\
Yes (Day 5) \\
Yes \\
Yes (Day 78) \\
Homozygous for pathogenic \\
variant C.1062+5G $>$ A (p.?) \\
in FAH-gene \\
Day 1 (14 hours of age) \\
2.5 mmol/L (45 mg/dL) \\
31.3 mU/L \\
Not done \\
Not done \\
Not done \\
1.62 mmol/L \\
Day 12 \\
$5-12$ mg/kg/day \\
3 months \\
- \\
- \\
Yes (on Day 84 ) \\
age of 13 weeks \\
\hline
\end{tabular}




\section{Case 2}

The second patient was the brother of Case 1 . He was born at 36 weeks of gestation and was screened for TT1 at $24 \mathrm{~h}$ after birth due to a positive family history of TT1. Biochemical parameters were suggestive of TT1 and genetic analysis confirmed the presence of the same homozygous mutation which was identified in his sister. He was treated with a tyrosine- and phenylalanine-restricted diet and NTBC since the first week of his life. He also had episodes of hypoglycaemia in the neonatal period, which initially were related to liver derangement due to TT1. However, despite improvement in his liver function, he was not able to maintain his BG. Hypoglycaemia screen confirmed the diagnosis of HH (Table 1) which was well controlled on the combination of diazoxide $(3 \mathrm{mg} / \mathrm{kg} /$ day) and chlorothiazide ( $7 \mathrm{mg} / \mathrm{kg} /$ day), which led to ageappropriate fasting tolerance without any supplemental carbohydrates. His treatment was discontinued after 6 months when he tolerated a $12 \mathrm{~h}$ fast, off diazoxide.

\section{Case 3}

The third patient was male, born in Germany at $36+4$ weeks of gestation to consanguineous parents of Syrian origin. Family history revealed that an older sibling passed away at the age of 11 years due to unknown liver disease in Syria and another sibling has been diagnosed with prune belly syndrome. Our patient had a complicated neonatal course with: (i) suspected sepsis requiring antibiotics with negative blood cultures and inflammatory markers, (ii) severe coagulopathy managed with repeated fresh frozen plasma (FFP), antithrombin III, vitamin $\mathrm{K}$ and platelet transfusions, (iii) cholestasis and conjugated hyperbilirubinemia, (iv) recurrent hypoglycaemia from as early as 14 hours of life. Initially, he required intravenous glucose with a glucose infusion rate of up to $12.5 \mathrm{mg} /$ $\mathrm{kg} / \mathrm{min}$ to maintain euglycaemia. Hypoglycaemia screen revealed the presence of insulin confirming the diagnosis of $\mathrm{HH}$ (Table 1). Therefore he was commenced on diazoxide initially at $5 \mathrm{mg} / \mathrm{kg} / \mathrm{day}$, which gradually was increased up to $12 \mathrm{mg} / \mathrm{kg} /$ day in order to stabilise his BG.

Tyrosinaemia type I was suspected early due to high tyrosine (180 $\mu \mathrm{mol} / \mathrm{L})$ and succinylacetone (6.99 $\mu \mathrm{mol} / \mathrm{L})$ levels on neonatal screening. Genetic testing confirmed the diagnosis and he was found to be homozygous for the pathogenic variant c.1062+5G $>A$ in the fumarylacetoacetate hydrolase (FAH) gene. Hence, he was commenced on a low tyrosine/phenylalanine diet and NTBC, with no side effects and was discharged home on the above treatment along with diazoxide. At 12 weeks of age, he was readmitted with signs of pulmonary hypertension requiring continuous positive airway pressure (CPAP), sildenafil and furosemide. Diazoxide was immediately discontinued as the perceived cause for his pulmonary hypertension (4). Following discontinuation of diazoxide his BG remained stable. A week later he tolerated a $7 \mathrm{~h}$-fast off diazoxide. Currently, at the age of 9 months he remains euglycaemic off diazoxide and with no signs of pulmonary hypertension. Treatment with sildenafil was discontinued and he is currently only on Lisinopril $0.027 \mathrm{mg} / \mathrm{kg} /$ day and NTBC.

\section{Discussion}

We describe three patients with TT1 and HH. All patients presented with liver failure in their first days of life. Biochemical and genetic testing confirmed the diagnosis in all patients. Besides the typical TT1 associated problems all patients repeatedly presented with episodes of hypoglycaemia persisting even after liver function improved. Detectable insulin levels at the time of real hypoglycaemia confirmed the diagnosis of $\mathrm{HH}$.

Hypoglycaemia in children with TT1 has already been described in early reports of tyrosinaemia. In 1967 Perry et al. described three patients with TT1 who suffered from hypoglycaemia (5). Histological analysis of the pancreas in children with tyrosinaemia revealed marked hypertrophy and increase in the number of the islets of Langerhans $(5,6)$. However, these histological abnormalities in the pancreas are not consistently seen in patients with TT1 (7). On the basis of laboratory findings and clinical observations of three patients with tyrosinaemia, SassKortsak et al. suggested that hypoglycaemia may occur due to a secondary impairment of gluconeogenesis and insufficient stores of liver glycogen (8). Baumann et al. (3) suspected that tyrosinaemia-specific metabolites could affect intracellular ATP homeostasis in beta cells, inducing inappropriate insulin secretion similar to that caused by glucose influx. However, the pathomechanism of hyperinsulinism in children with TT1 has not been elucidated yet, and it remains unclear why only a small number of patients with TT1 suffer from $\mathrm{HH}$.

Diazoxide, the first line drug for the treatment of congenital hyperinsulinism (9), was started in all patients and led to euglycaemia; however, the required dose was quite variable between 3 and $12 \mathrm{mg} / \mathrm{kg} /$ day. Unfortunately, patient 3 developed pulmonary hypertension at 3 months of age. This might be related to the fact that he was treated with a fairly high dose 
of diazoxide without concomitant diuretic therapy. This severe side effect is seen in approximately $2.4 \%$ of all children treated with diazoxide (9). Treatment with diazoxide was discontinued and he remained euglycaemic since then. Responsiveness to diazoxide in children with TT1 and HH was also described in three patients reported by Baumann et al. in 2005 (3). As opposed to our patients who were treated with diazoxide for 3-8 months the patients reported by Baumann et al. needed treatment for 9-34 months (3), however, remission might also have occurred earlier as diazoxide withdrawal was tried quite late. The age of onset of hypoglycaemia differs widely between the patients. As opposed to our three patients, who were diagnosed with their first hypoglycaemic episodes between 1 and 14 days of life, the three patients reported by Baumann et al. were diagnosed with their first hypoglycaemic episode later, between 21 and 84 days of life (3). It remains unclear if these children had undiagnosed hypoglycaemic episodes earlier because symptoms of hypoglycaemia can be subtle in neonates and might be easily overseen. It is well known that adverse neurodevelopment is reported in $26-48 \%$ of patients with recurrent hyperinsulinaemic hypoglycaemia (10). Children with TT1 have an increased risk for neurocognitive problems, including a lower IQ and abnormal motor skills (11), which should not be aggravated by a delayed diagnosis or inadequate treatment of $\mathrm{HH}$, especially because treatment with diazoxide is effective in children with TT1 and HH.

\section{Declaration of interest}

The authors declare that there is no conflict of interest that could be perceived as prejudicing the impartiality of the research reported.

\section{Funding}

This research did not receive any specific grant from any funding agency in the public, commercial or not-for-profit sector.

\section{Patient consent}

Informed consent has been obtained from all patients.

\section{Author contribution statement}

Ellada Sotiridou and Henrike Hoermann contributed to writing and editing of the manuscript. Spyros Batzios, Sommayya Aftab, Antonia Dastamani, Sebastian Kummer and Pratik Shahwere involved in editing and revising the manuscript. Spyros Batzios, Louise Doodson, Eva Thimm, Sebastian Kummerand Pratik Shah coordinated the clinical care of patients. Ellada Sotiridou and Henrike Hoermann have contributed equally as joint first authors. Sebastian Kummer and Pratik Shah have contributed equally as joint senior/corresponding authors.

\section{References}

1 Chinsky JM, Singh R, Ficicioglu C, van Karnebeek CDM, Grompe M, Mitchell G, Waisbren SE, Gucsavas-Calikoglu M, Wasserstein MP, Coakley K, et al. Diagnosis and treatment of tyrosinemia type I: a US and Canadian consensus group review and recommendations. Genetics in Medicine 201719 12. (https://doi.org/10.1038/ $\operatorname{gim} .2017 .101)$

2 Mannion MA, Smith A, Mayne P \& Monavari AA. Type 1 tyrosinaemia. Irish Medical Journal 2016109426.

3 Baumann U, Preece MA, Green A, Kelly DA \& McKiernan PJ. Hyperinsulinism in tyrosinaemia type I. Journal of Inherited Metabolic Disease 200528 131-135. (https://doi.org/10.1007/s10545-005-5517-1)

4 Chen SC, Dastamani A, Pintus D, Yau D, Aftab S, Bath L, Swinburne C, Hunter L, Giardini A, Christov G, et al. Diazoxideinduced pulmonary hypertension in hyperinsulinaemic hypoglycaemia: recommendations from a multicentre study in the United Kingdom. Clinical Endocrinology 201991 770-775. (https:// doi.org/10.1111/cen.14096)

5 Perry TL. Tyrosinemia associated with hypermethioninemia and islet cell hyperplasia. Canadian Medical Association Journal 196797 1067-1075.

6 Halvorsen S, Pande H, Loken AC \& Gjessing LR. Tyrosinosis. A study of 6 cases. Archives of Disease in Childhood 196641 238-249. (https:// doi.org/10.1136/adc.41.217.238)

7 Russo P \& O'Regan S. Visceral pathology of hereditary tyrosinemia type I. American Journal of Human Genetics 199047 317-324.

8 Sass-Kortsak A, Ficici S, Paunier L, Kooh SW, Fraser D \& Jackson SH. Secondary metabolic derangements in patients with tyrosyluria. Canadian Medical Association Journal 196797 1079-1083.

9 De Cosio AP \& Thornton P. Current and emerging agents for the treatment of hypoglycemia in patients with congenital hyperinsulinism. Pediatric Drugs 201921 123-136. (https://doi. org/10.1007/s40272-019-00334-w)

10 Banerjee I, Salomon-Estebanez M, Shah P, Nicholson J, Cosgrove KE \& Dunne MJ. Therapies and outcomes of congenital hyperinsulinism-induced hypoglycaemia. Diabetic Medicine 201936 9-21. (https://doi.org/10.1111/dme.13823)

11 Van Ginkel WG, Rodenburg IL, Harding CO, Hollak CEM, HeinerFokkema MR \& van Spronsen FJ. Long-term outcomes and practical considerations in the pharmacological management of tyrosinemia Type 1. Pediatric Drugs 201921 413-426. (https://doi.org/10.1007/ s40272-019-00364-4)

Received in final form 29 October 2020

Accepted 26 November 2020 